\title{
PRINCIPAL SOLUTIONS OF POSITIVE LINEAR HAMILTONIAN SYSTEMS
}

\author{
DON HINTON
}

(Received 28 July 1975)

\begin{abstract}
The Hamiltonian system $Y^{\prime}=B Y+C Z, Z^{\prime}=-A Y-B^{*} Z$ is considered where the coefficients are continuous on $I=[a, \infty), C=C^{*} \geqq 0$, and $A=A^{*} \leqq 0$. A solution $(Y, Z)$ satisfying $Y^{*} Z=Z^{*} Y$ is defined to be principal (coprincipal) provided that (i) $Y^{-1}$ exists on $I$ $\left(Z^{\prime}\right.$ exists on $I$ ) and (ii) $\left[\int_{a}^{1} Y^{-1} C Y^{*-1}\right]^{1} \rightarrow 0$ as $t \rightarrow \infty\left(\left[\int_{a}^{\prime}-Z^{-1} A Z^{*-1}\right]^{-1} \rightarrow 0\right.$ as $t \rightarrow \infty$ ). Three conditions are given which are separately equivalent to the condition that a solution is principal iff it is coprincipal. For a self-adjoint scalar operator $L$ of order $2 n$, this problem is related to the deficiency index problem and to a problem of Anderson and Lazer (1970) which concerns the number of linearly independent solutions of $L(y)=0$ satisfying $y^{(k)} \in$ $\mathscr{L}_{2}(a, x)(k=0, \cdots, n)$.
\end{abstract}

We consider here the Hamiltonian system

$$
\begin{aligned}
& y^{\prime}=B(t) y+C(t) z, \quad a \leqq t<\infty, \\
& z^{\prime}=-A(t) y-B^{*}(t) z
\end{aligned}
$$

where $y$ and $z$ are $n$-vectors; the corresponding matrix equation is

$$
Y^{\prime}=B(t) Y+C(t) Z
$$

$$
Z^{\prime}=-A(t) Y-B^{*}(t) Z
$$

where $Y$ and $Z$ are $n \times n$ matrix functions. The conditions that we will sometimes impose on the system (1) are:

$\left(\mathrm{P}_{0}\right) A, B$, and $C$ are continuous, complex-valued matrix functions on $[a, \infty)$ with $C=C^{*}$ and $A=A^{*}$.

$\left(\mathrm{P}_{1}\right)$ For each $t \in[a, \infty), C(t) \geqq 0$, i.e., $\eta^{*} C(t) \eta \geqq 0$ for all vectors $\eta$.

$\left(\mathrm{P}_{2}\right)$ For each $t \in[a, \infty),-A(t) \geqq 0$.

$\left(\mathrm{P}_{3}\right)$ If $(y, z)$ is a solution of (1) on an interval $I$ and $y \equiv 0$ on $I$, then $z \equiv 0$ on $I$. 
$\left(\mathrm{P}_{4}\right)$ If $(y, z)$ is a solution of $(1)$ on an interval $I$ and $z \equiv 0$ on $I$, then $y \equiv 0$ on $I$.

$\left(\mathrm{P}_{5}\right)$ If $(y, z)$ is a solution of $(1)$ such that

$$
\limsup _{r \rightarrow \infty} \int_{a}^{t}\left[-y^{*} A y+z^{*} C z\right]<\infty,
$$

then $\left(y^{*} z\right)(t) \rightarrow 0$ as $t \rightarrow \infty$.

Under $\left(P_{0}\right)$ it follows that if $\left(Y_{1}, Z_{1}\right)$ and $\left(Y_{2}, Z_{2}\right)$ are solutions of $(2)$, then $Y_{1}^{*} Z_{2}-Z_{1}^{*} Y_{2}$ is a constant matrix. A solution $(Y, Z)$ of $(2)$ is said to be isotropic or self-conjoined if the constant matrix $Y^{*} Z-Z^{*} Y$ is zero. An isotropic solution $(Y, Z)$ of $(2)$ is said to be principal (coprincipal) on an interval $I=[c, b)$ provided that:

(i) $Y^{-1}$ exists on $I\left(Z^{-1}\right.$ exists on $\left.I\right)$.

(ii) $\left[\int_{t} Y^{-1} C Y^{*-1}\right]^{-1} \rightarrow 0$ as $t \rightarrow b\left(\left[\int_{c}^{t}-Z^{-1} A Z^{*-1}\right]^{-1} \rightarrow 0\right.$ as $\left.t \rightarrow b\right)$.

Properties of principal solutions are given in Chapter 2 of Coppel (1971) or Chapter 7 of Reid (1971). Two important properties are: (i) $\left(\mathrm{P}_{0}\right),\left(\mathrm{P}_{1}\right),\left(\mathrm{P}_{3}\right)$ and the existence of an isotropic solution $(Y, Z)$ of $(2)$ with $Y^{-1}$ existing on $I=[c, b)$ imply that a principal solution exists on $I$. (ii) $\left(\mathrm{P}_{0}\right)-\left(\mathrm{P}_{3}\right)$ imply that a principal solution exists on $[a, \infty)$. By symmetry, similar conclusions hold for coprincipal solutions; for example, $\left(\mathrm{P}_{0}\right)-\left(\mathrm{P}_{2}\right)$ and $\left(\mathrm{P}_{4}\right)$ imply that a coprincipal solution exists on $[a, \infty)$. We note also that $\left(\mathrm{P}_{0}\right)-\left(\mathrm{P}_{3}\right)$ imply uniqueness of a principal solution $(Y, Z)$ in the sense that if $\left(Y_{1}, Z_{1}\right)$ is also a principal solution, then $\left(Y_{1}, Z_{1}\right)=(Y K, Z K)$ for some constant non-singular matrix $K$.

The main result of this paper is Theorem 3 which gives conditions under which the concepts of principal and coprincipal solutions coincide. This problem has been investigated recently by Ahlbrandt $(1972,1976)$. Ahlbrandt (1972) considers the system $(2)$ when $\left(P_{0}\right),\left(P_{1}\right),\left(P_{3}\right),\left(P_{4}\right)$, and $A(t) \geqq 0$ hold and gives a condition for the equivalence of principal and coprincipal solutions (Theorem 4.1). We consider here only the case $A(t) \leqq 0$. Ahlbrandt (1976) considers (2) when $A(t)$ is of constant sign. Conditions are given which are equivalent to the statement that a solution of (2) is principal if and only if it is coprincipal. The conditions of Ahlbrandt are motivated by oscillation theory whereas conditions (i) and (iv) of our Theorem 3 are motivated by the limit-point theory of differential equations. This connection is discussed following Theorem 3. There is some overlap between condition (iii) of Theorem 3 and the conditions of Ahlbrandt (1976) although Ahlbrandt does not explicitly give condition (iii).

The following example is pertinent to the connection with limit point theory and also gives an example where the notions of principal and coprincipal are different. 
Let $p$ be a positive continuous function on $[0, \infty)$ with $\int_{0}^{\infty} p d t<\infty$. The scalar equation $\left(y^{\prime} / p\right)^{\prime}=p y$ has the system formulation

$$
y^{\prime}=p z, \quad z^{\prime}=p y .
$$

Clearly, $\left(\mathrm{P}_{0}\right)-\left(\mathrm{P}_{4}\right)$ hold. One solution of (3) is

$$
y_{0}(t)=z_{0}(t)=\exp \left[-\int_{t}^{\infty} p\right]
$$

A linearly independent solution is given by

$$
\begin{aligned}
& y_{1}(t)=y_{0}(t) \int_{1}^{\infty} p / y_{0}^{2} \\
& z_{1}(t)=y_{0}(t) \int_{t}^{\infty} p / y_{0}^{2}-1 / y_{0}(t) .
\end{aligned}
$$

It may be verified that $\left(y_{1}, z_{1}\right)$ is a principal solution of (3). However, $\left(y_{1}, z_{1}\right)$ is not coprincipal since $z_{1}(t) \rightarrow-1$ as $t \rightarrow \infty$.

A differentiation shows that if $(Y, Z)$ is an isotropic solution of (2) and $\left(\mathrm{P}_{0}\right)$ holds, then

$$
\left(Y^{*} Z\right)^{\prime}=\left(Z Y^{*}\right)^{\prime}=-Y^{*} A Y+Z^{*} C Z,
$$

and at any $t$ where $\left(Y^{*} Z\right)^{-1}$ exists,

$$
-\left[\left(Y^{*} Z\right)^{-1}\right]^{\prime}=Y^{-1} C Y^{*-1}-Z^{-1} A Z^{*-1} \text {. }
$$

TheOREM 1. Suppose $\left(\mathrm{P}_{0}\right)-\left(\mathrm{P}_{3}\right)$ hold, $(Y, Z)$ is a principal solution of (2) on $I=[c, b)$, and $W=Z Y^{-1}$. Then

(i) $\operatorname{limit}_{t \rightarrow b} \int_{t}\left[-Y^{*} A Y+Z^{*} C Z\right]$ exists and is finite.

(ii) If also $\left(\mathrm{P}_{4}\right)$ holds, then $W(t)<0, c \leqq t<b$, and $Y^{*} Z \rightarrow 0$ as $t \rightarrow b$.

Proof. Since $\left(\mathrm{P}_{0}\right)-\left(\mathrm{P}_{3}\right)$ imply that $W(t) \leq 0$ [p. 55 of Coppel], we may integrate (4) to obtain

$$
\int_{c}^{t}\left[-Y^{*} A Y+Z^{*} C Z\right]=\left.Y^{*} Z\right|_{\epsilon}
$$

(6)

$$
=\left.Y^{*} W Y\right|_{\downarrow} \leqq-\left[Y^{*} W Y\right](c) .
$$

Since $-A \geqq 0$ and $C \geqq 0$, the integral in (6) is non-decreasing and bounded above by a hermitian matrix; hence (i) holds.

Now $W(t) \leqq 0$ and $Y^{-1}$ exists; hence $W(t)<0$ if $Z^{-1}$ exists on $I$. Suppose to the contrary $Z\left(t_{0}\right) \eta=0$ for some $\eta \neq 0$ and $c \leqq t_{0}<b$. Then for $t>t_{0}$, 


$$
\begin{aligned}
0 \leqq \int_{t_{0}}^{t} \eta^{*}\left[-Y^{*} A Y+Z^{*} C Z\right] \eta & =\eta^{*}\left[\left(Y^{*} Z\right)(t)-\left(Y^{*} Z\right)\left(t_{0}\right)\right] \eta \\
& =\eta^{*}\left(Y^{*} W Y\right)(t) \eta \leqq 0
\end{aligned}
$$

thus $(Y \eta)^{*} A(Y \eta)=(Z \eta)^{*} C(Z \eta)=0$ on $\left[t_{0}, t\right)$ which implies that $A Y \eta=$ $C Z \eta=0$ on $\left[t_{0}, t\right]$ since $C \geqq 0$ and $-A \geqq 0$. Therefore the vectors $(y, z)=$ $(Y \eta, Z \eta)$ satisfy $y^{\prime}=B y, z^{\prime}=-B^{*} z$ and $z\left(t_{0}\right)=0$ which implies $z \equiv 0$ on $\left[t_{0}, t\right]$. By $\left(\mathrm{P}_{4}\right), y=Y \eta \equiv 0$ on $\left[t_{0}, t\right]$ which contradicts $Y$ being non-singular; hence $W(t)<0$ on $I$. Note also that $Y^{*} Z=Y^{*} W Y<0$.

Since $W(t)<0, Z$ is also invertiable, and we have by (5).

$$
\begin{aligned}
-\left(Y^{*} Z\right)^{-1}(t) & \geqq\left(Y^{*} Z\right)^{-1}(c)-\left(Y^{*} Z\right)^{-1}(t) \\
& =\int_{c}^{l}\left[Y^{-1} C Y^{*-1}-Z^{-1} A Z^{*-1}\right] \geqq \int_{c}^{l} Y^{-1} C Y^{*-1} .
\end{aligned}
$$

This inequality and the definition of principal solutions yield that $Y^{*} Z \rightarrow 0$ as $t \rightarrow b$.

It is possible that $W=0$ in Theorem 1 as is shown by the scalar system $y^{\prime}=z, z^{\prime}=0$ which has as principal solution $(y, z)=(1,0)$.

We note from the above proof that if $(Y, Z)$ is an isotropic solution of (2) and $\left(\mathrm{P}_{0}\right)-\left(\mathrm{P}_{4}\right)$ hold, then: (i) $Y^{*} Z$ is strictly increasing on $I=[c, b)$ if $Y^{-1}$ and $Z^{-1}$ exist on $I$ (integrate (5) and use the fact that $\int_{t} Y^{-1} C Y^{*-1}$ is strictly increasing [p. 38 of Coppel]), and (ii) if for some $t_{0}$, one of $Y\left(t_{0}\right)$ and $Z\left(t_{0}\right)$ is zero and the other is non-singular, then $\left(Y^{*} Z\right)(t)>0$ for $t>t_{0}$ and $\left(Y^{*} Z\right)(t)<0$ for $t<t_{0}$. To establish this last fact it follows from (4) that if $\left(Y^{*} Z\right)(t) \eta=0$ for some $t \neq t_{0}$ and $\eta \neq 0$, then

$$
\int_{t_{0}}^{t} \eta^{*}\left[-Y^{*} A Y+Z^{*} C Z\right] \eta=0
$$

and we may repeat the argument following (7) to obtain a contradiction. Since $Y^{*} Z$ is non-decreasing, this completes the proof.

By symmetry, conclusion (i) of Theorem 1 holds for a coprincipal solution if $\left(\mathrm{P}_{0}\right)-\left(\mathrm{P}_{2}\right)$ and $\left(\mathrm{P}_{4}\right)$ hold, and conclusion (ii) holds for a coprincipal solution if $\left(\mathrm{P}_{0}\right)-\left(\mathrm{P}_{4}\right)$ hold.

THEOREM 2. Suppose $\left(\mathrm{P}_{0}\right)-\left(\mathrm{P}_{4}\right)$ hold, $\left(Y_{1}, Z_{1}\right)$ is a principal solution of (2) on $I=[c, b)$, and $\left(Y_{2}, Z_{2}\right)$ is a coprincipal solution of (2) on $I$. Then

(i) $Z_{1} Y_{1}^{-1} \leqq Z_{2} Y_{2}^{-1}$ on $\mathrm{I}$.

(ii) If $(Y, Z)$ is an isotropic solution of (2) satisfying $Z_{1}(c) Y_{1}^{-1}(c) \leqq$ $Z(c) Y^{-1}(c) \leqq Z_{2}(c) Y_{2}^{-1}(c)$, then $Y^{-1}$ and $Z^{-1}$ exist on $I$. 
Proof. By Theorem $1, Z_{1}^{-1}$ and $Y_{2}^{-1}$ exist on $I$. Let $W_{1}=Z_{1} Y_{1}^{-1}$ and $W_{2}=Z_{2} Y_{2}^{-1}$. Since both $W_{1}$ and $W_{2}$ are symmetric solutions on $I$ of the matrix Riccati equation

$$
W^{\prime}+A(t)+W B(t)+B^{*}(t) W+W C(t) W=0
$$

we must have (i) by Theorem 8, p. 54 of Coppel.

Let $W_{3}=Z Y^{-1}$; then $W_{3}$ is a solution of (8) and again by Coppel $W_{3}$ exists on $I$ which implies $Y^{-1}$ exists on $I$. Since $W_{3}^{-1}(c)=Y(c) Z^{-1}(c) \geqq$ $Y_{2}(c) Z_{2}^{-1}(c)$, and $W_{3}^{-1}$ and $Y_{2} Z_{2}^{-1}$ satisfy the Riccati equation

$$
W^{\prime}-C-W B^{*}-B W-W A W=0,
$$

we again apply Theorem 8 of Coppel to conclude that $W_{3}^{-1}$ exists on $I$; hence $Z^{-1}$ exists on $I$.

THEOREM 3. Suppose $\left(\mathrm{P}_{0}\right)-\left(\mathrm{P}_{4}\right)$ hold. Then the following are equivalent.

(i) $\left(\mathrm{P}_{5}\right)$

(ii) An isotropic solution ( $Y, Z)$ of (2) is principal if and only if it is coprincipal (on $[a, \infty)$ ).

(iii) If $(Y, Z)$ is an isotropic solution of (2) with $\left(Y^{*} Z\right)(c)>0, c \geqq a$, then $\left(Y^{*} Z\right)^{-1} \rightarrow 0$ as $t \rightarrow \infty$.

(iv) $\operatorname{dim} Q=n$ where $Q$ is the set of all solutions $(y, z)$ of (1) such that $\int_{a}^{\infty}\left[-y^{*} A y+z^{*} C z\right]<\infty$.

Proof. (i) $\rightarrow$ (ii). Let $\left(Y_{1}, Z_{1}\right)$ be a principal solution of $(2)$ on $[a, \infty)$ and $\left(Y_{2}, Z_{2}\right)$ be a coprincipal solution of $(2)$ on $[a, \infty)$. Define the solution $\left(Y_{3}, Z_{3}\right)$ of (2) by

$$
\begin{aligned}
& Y_{3}(t)=Y_{1}(t) Y_{1}^{-1}(a)-Y_{2}(t) Y_{2}^{-1}(a) \\
& Z_{3}(t)=Z_{1}(t) Y_{1}^{-1}(a)-Z_{2}(t) Y_{2}^{-1}(a)
\end{aligned}
$$

By Theorem 1,

$$
\int_{a}^{\infty}\left[Y_{i}^{*} A Y_{i}+Z_{i}^{*} C Z_{i}\right]<\infty
$$

for $i=1,2$; hence (9) holds for $i=3$. By $\left(\mathrm{P}_{5}\right)$, this implies diagonal $Y_{3}^{*} Z_{3} \rightarrow 0$ as $t \rightarrow \infty$. Now $Y_{3}(a)=0$ and (4) imply that $\left(Y^{*} Z\right)(t) \geqq 0$; hence $Y_{3}^{*} Z_{3} \rightarrow 0$ as $t \rightarrow \infty$. Therefore

$$
0=-\left(Y_{3}^{*} Z_{3}\right)(a)=\int_{a}^{\infty}\left[-Y_{3}^{*} A Y_{3}+Z_{3}^{*} C Z_{3}\right]
$$

from which we conclude that $A Y_{3} \equiv 0$ and $C Z_{3} \equiv 0$. The function $Y_{3}$ then satisfies $Y_{3}^{\prime}=B(t) Y_{3}, Y_{3}(a)=0$ which yields $Y_{3}=0$ or

$$
Y_{2}=Y_{1} Y_{1}^{-1}(c) Y_{2}(c) \text {. }
$$


Therefore $\left[\int_{c}^{t} Y_{2}^{-1} C Y_{2}^{*-1}\right]^{-1} \rightarrow 0$ as $t \rightarrow b$ or $\left(Y_{2}, Z_{2}\right)$ is principal. Since principal and coprincipal solutions are unique up to constant multiples, this completes the proof.

(ii) $\rightarrow$ (iii). Let $\left(Y_{0}, Z_{0}\right)$ be a principal solution of (2) on $[c, \infty)$, and let $(Y, Z)$ be an isotropic solution with $\left(Y^{*} Z\right)(c)>0$. Now $Y^{*} Z$ is nondecreasing so that $Y^{*} Z$ is invertiable on $[c, \infty)$. Define

$$
\begin{array}{ll}
s(t)=\int_{c}^{t} Y^{-1} C Y^{*-1}, & s_{0}(t)=\int_{c}^{t} Y_{0}^{-1} C Y_{0}^{*-1}, \\
\hat{s}(t)=\int_{c}^{t}-Z^{-1} A Z^{*-1}, & \hat{s}_{0}(t)=\int_{c}^{t}-Z_{0}^{-1} A Z_{0}^{*-1} .
\end{array}
$$

By applying Proposition 1 of Coppel, p. 35, to (2) and also to (2) with the order of the equations reversed, we obtain

$$
\begin{aligned}
& Y(t)=Y_{0}(t)\left[M_{1}+s_{0}(t) N_{1}\right], \\
& Z(t)=Z_{0}(t)\left[M_{2}+\hat{s}_{0}(t) N_{2}\right],
\end{aligned}
$$

where $Y(c)=Y_{0}(c) M_{1}, Z(c)=Z_{0}(c) M_{2}$, and

$$
N_{1}=Y_{0}^{*}(c) Z(c)-Z_{0}^{*}(x) Y(c)=-\left[Z_{0}^{*}(c) Y(c)-Y_{0}^{*}(c) Z(c)\right]=-N_{2} .
$$

Also from Coppel (1971)

$$
\begin{aligned}
& s(t)=\left[M_{1}+s_{0}(t) N_{1}\right]^{-1} s_{0}(t) M_{1}^{*-1}, \\
& \hat{s}(t)=\left[M_{2}-\hat{s}_{0}(t) N_{1}\right]^{-1} \hat{s}_{0}(t) M_{2}^{*-1} .
\end{aligned}
$$

Recalling that the definition of principal solution gives that $s_{0}^{-1}(t)$ and $\hat{s}_{0}^{-1}$ tend to 0 as $t \rightarrow \infty$, we then have that (note that $N_{1}^{-1}$ exists since it is sufficient that $Y_{0}(c)=Y(c)=E$ in which case $Z_{0}(c)<0, Z(c)>0$, and $\left.N_{1}>0\right)$

$$
\operatorname{limit}_{t \rightarrow \infty}[s(t)+\hat{s}(t)]=N_{1}^{-1} M_{1}^{*-1}-N_{1}^{-1} M_{2}^{*-1} .
$$

Now by (5),

$$
\left(Y^{*} Z\right)^{-1}(t)=\left(Y^{*} Z\right)^{-1}(c)-[s(t)+\hat{s}(t)]
$$

hence using the above relations and the fact that $(Y, Z)$ is isotropic, we obtain

$$
\begin{aligned}
\operatorname{limit}_{i \rightarrow \infty} & \left(Y^{*} Z\right)^{-1}(t)=\left(Y^{*} Z\right)^{-1}(c)-N_{1}^{-1} M_{1}^{*-1}+N_{1}^{-1} M_{2}^{*-1} \\
& =N_{1}^{-1}\left[\left(Y_{0}^{*}(c) Z(c)-Z_{0}^{*}(c) Y(c)\right)\left(Y^{*} Z\right)^{-1}(c)-M_{1}^{*-1}+M_{2}^{*-1}\right] \\
& =N_{1}^{-1}\left[Y_{0}^{*}(c) Y^{*-1}(c)-Z_{0}^{*}(c) Z^{*-1}(c)-M_{1}^{*-1}+M_{2}^{*-1}\right] \\
& =N_{1}^{-1}\left[Y_{0}^{*}(c)\left(M_{1}^{*} Y_{0}^{*}(c)\right)^{-1}-Z_{0}^{*}(c)\left(M_{2}^{*} Z_{0}^{*}(c)\right)^{-1}-M_{1}^{*-1}+M_{2}^{*-1}\right] \\
& =N_{1}^{-1}\left[M_{1}^{*-1}-M_{2}^{*-1}-M_{1}^{*-1}+M_{2}^{*-1}\right]=0 .
\end{aligned}
$$


(iii) $\rightarrow$ (iv). Define the isotropic solution $(Y, Z)$ of (2) by the initial conditions $Y(a)=0, Z(a)=E$. Then $\left(Y^{*} Z\right)(t)>0$ for $t>a$ which by (iii) implies $\left(Y^{*} Z\right)^{-1}(t) \rightarrow 0$ as $t \rightarrow \infty$. Suppose now $\operatorname{dim} Q>n$. Hence there is a member $(y, z)$ in $Q$ with $y(a)=0, y \neq 0$. This implies that $y=Y \eta, z=Z \eta$ where $\eta=z(a) \neq 0$. By (4) we have for $t>a$,

$$
0<\eta^{*}\left(Y^{*} Z\right)(t) \eta=\left(y^{*} z\right)(t)=\int_{a}^{t}\left[-y^{*} A y+z^{*} C z\right]
$$

which implies that $\left(y^{*} z\right)(t)$ tends to a positive constant as $t \rightarrow \infty$. Now for a positive matrix $T$ and a vector $v$,

$$
\begin{aligned}
v^{*} v=\langle v, v\rangle & =\left\langle T v, T^{-1} v\right\rangle \leqq\langle T v, v\rangle^{1 / 2}\left\langle T T^{-1} v, T^{-1} v\right\rangle^{1 / 2} \\
& =\langle T v, v\rangle^{1 / 2}\left\langle T^{-1} v, v\right\rangle^{1 / 2}
\end{aligned}
$$

Using this inequality with $v=\eta$ and $T=\left(Y^{*} Z\right)(t)$ contradicts the fact that $\left(Y^{*} Z\right)^{-1}(t) \rightarrow 0$ as $t \rightarrow \infty$; hence $\operatorname{dim} Q=n$.

(iv) $\rightarrow$ (i). Let $(Y, Z)$ be a principal solution of $(2)$ on $[a, \infty)$. If $\left(y_{i}, z_{i}\right)$ are the $i$ th columns of $Y, Z$ respectively, then part (i) of Theorem 1 implies $\left(y_{i}, z_{i}\right) \in Q$. Since $Q$ is $n$ dimensional and the $n$ columns of $Y, Z$ are linearly independent solutions of (1), the members $(y, z)$ of $Q$ are of the form $y=Y \eta$, $z=Z \eta$ for some constant vector $\eta$. Therefore by part (ii) of Theorem 1,

$$
\operatorname{limit}_{i \rightarrow \infty} y^{*} z=\operatorname{limit}_{i \rightarrow \infty} \eta^{*}\left(Y^{*} Z\right) \eta=0 .
$$

The scalar equation

$$
L(x)=\sum_{i=0}^{n}(-1)^{i}\left(p_{i} x^{(i)}\right)^{(i)}=0, \quad a \leqq t<\infty,
$$

where the $p_{i}$ are real, continuous functions with $p_{n}>0$ can be put into the form (1). Let the quasi-derivatives $x^{[i]}, i=n, \cdots, 2 n$, be defined by: $x^{|n|}=$ $p_{n} x^{(n)}, \quad x^{[n+i+1]}=-\left(x^{[n+i]}\right)^{\prime}+p_{n-i-1} x^{(n-i-1)} \quad(i=0, \cdots, n-1)$. Then $L(y)=$ $x^{(2 n)}=0$ has the form (1) where

$$
A_{i j}=\left\{\begin{array}{cc}
0, & i \neq j \\
-p_{i-1}, & i=j,
\end{array} \quad B_{i j}=\left\{\begin{array}{ll}
0, & j \neq i+1 \\
1, & j=i+1,
\end{array} \quad C_{i j}=\left\{\begin{array}{cc}
0, & i \neq n, \quad j \neq n \\
p_{n}^{-1}, & i=j=n .
\end{array}\right.\right.\right.
$$

Clearly $\left(\mathrm{P}_{0}\right),\left(\mathrm{P}_{1}\right)$, and $\left(\mathrm{P}_{3}\right)$ hold. $\left(\mathrm{P}_{4}\right)$ holds if $p_{0}$ does not vanish identically on any interval. The condition $\left(\mathrm{P}_{2}\right)$ is equivalent to $p_{i} \geqq 0,0 \leqq i \leqq n-1$. With $Q$ as in Theorem 3 and each $p_{i} \geqq 0,(y, z) \in Q$ if and only if 


$$
\int_{a}^{\infty}\left[p_{n}\left(x^{(n)}\right)^{2}+\cdots+p_{0} x^{2}\right]<\infty
$$

If $\quad p_{i} \geqq 0 \quad(i=1, \cdots, n-1) \quad$ and $\quad p_{0}(t) \geqq k>0$, then (12) implies $x \in \mathscr{L}_{2}[a, \infty)$.Thus if $L$ in (10) is limit point or limit- $n$ at infinity (which in this case is equivalent to $L(x)=0$ having $n$ linearly independent solutions in $\mathscr{L}_{2}[a, \infty)$ ), then $\operatorname{dim} Q=n$. For example $(10)$ is limit $n$ at infinity if (see Hinton (1972)) $p_{n}(t)=t^{\alpha}(\alpha \leqq 2 n), p_{0}(t) \geqq k>0$, and for $i=1, \cdots, n-1$,

$$
0 \leqq p_{i}(t) \leqq k_{i} t^{\nu_{i}}, \quad \gamma_{i}=[2(n-i)+\alpha(2 i-1)] /(2 n-1), \quad k_{\mathrm{i}}>0 .
$$

As a further example we will prove that $\operatorname{dim} Q=n=2$ for

$$
L_{4}(x)=x^{(i v)}-\left(q x^{\prime}\right)^{\prime}+p x, \quad a \leqq t<\infty,
$$

where the real, continuous functions $q$ and $p$ satisfy $p(t) \geqq k>0, q(t) \geqq 0$, $q=q_{1}+q_{2}$ where $q_{1}^{\prime}(t) \geqq-k_{0} t$ and $\left|q_{2}(t)\right| \leqq k_{0} t, k_{0}>0$. Suppose $\operatorname{dim} Q>2$. Then using (12), we have a solution $x$ of $L_{4}(x)=0$ such that $x(a)=x^{\prime}(a)=0$ and

$$
\left.1=\int_{a}^{\infty}\left[\left(x^{\prime \prime}\right)^{2}+q\left(x^{\prime}\right)^{2}+p x^{2}\right]\right) d t
$$

After an integration by parts of

$$
\left\{-\left(x^{\prime \prime \prime}-q x^{\prime}\right) x+x^{\prime \prime} x^{\prime}\right\}^{\prime}=\left(x^{\prime \prime}\right)^{2}+q\left(x^{\prime}\right)^{2}+p x^{2},
$$

we obtain that

$$
1=\operatorname{limit}_{t \rightarrow \infty} \int_{a}^{t}\left[\left(x^{\prime \prime}\right)^{2}+q\left(x^{\prime}\right)^{2}+p x^{2}\right]\left(1-\frac{\tau}{t}\right) d \tau
$$

$$
=\operatorname{limit}_{t \rightarrow \infty} \frac{1}{t} \int_{a}^{t}\left[-\left(x^{\prime \prime \prime}-q x^{\prime}\right) x+x^{\prime \prime} x^{\prime}\right] d \tau .
$$

Now (13) implies $x, x^{\prime \prime} \in \mathscr{L}_{2}(a, \infty)$ which implies $x^{\prime} \in \mathscr{L}_{2}(a, \infty)$ and

$$
\operatorname{limit}_{t \rightarrow \infty} \frac{1}{t} \int_{a}^{t} x^{\prime \prime} x^{\prime} d \tau=0
$$

Now $L_{4}(x)=0$ and $x \in \mathscr{L}_{2}(a, \infty)$ yields that $x$ is oscillatory by Lemma 2 of Hinton (1973); hence for $x\left(t_{n}\right)=0, t_{n} \rightarrow \infty$ as $n \rightarrow \infty$, 


$$
-\frac{1}{t_{n}} \int_{a}^{t_{n}} x^{\prime \prime \prime} x d \tau=\frac{1}{t_{n}} \int_{a}^{t_{n}} x^{\prime \prime} x^{\prime} d \tau \rightarrow 0 \quad \text { as } \quad n \rightarrow \infty .
$$

Using (15) and (16) in (14) yields

$$
1=\operatorname{limit}_{n \rightarrow \infty} \frac{1}{t_{n}} \int_{a}^{t_{n}}\left[q_{1}+q_{2}\right] x x^{\prime} d \tau .
$$

However, we have that

and

$$
\frac{1}{t_{n}} \int_{a}^{t_{n}} q_{1} x^{\prime} x d \tau=-\frac{1}{2 t_{n}} \int_{a}^{t_{n}} q_{1}^{\prime} x^{2} d \tau \leq \frac{k_{0}}{2 t_{n}} \int_{a}^{t_{n}} \tau x^{2} d \tau
$$

$$
\left|\frac{1}{t_{n}} \int_{a}^{t_{n}} q_{2} x x^{\prime} d \tau\right| \leqq \frac{k_{0}}{t_{n}} \int_{a}^{i_{n}} \tau\left|x x^{\prime}\right| d \tau .
$$

The right hand sides of these two inequalities tends to zero as $n \rightarrow \infty$ since $f \in \mathscr{L}_{1}(a, \infty)$ implies $t^{-1} \int_{a}^{t} \tau|f(\tau)| d \tau \rightarrow 0$ as $t \rightarrow \infty$. This is a contradiction to (17) and $\operatorname{dim} Q=2$ is established.

Thus it may be conjectured that the principal and coprincipal solutions of (10) coincide under the conditions $p_{n}>0, p_{i} \geqq 0$ for $i=1, \cdots, n-1$, and $p_{0} \geqq k>0$. That the condition $p_{0} \geqq k>0$ cannot be replaced by $p_{0} \geqq 0$ is shown by our first example.

A related problem to showing $\operatorname{dim} Q=n$ was considered by Anderson and Lazer (1970). Assuming the $p_{i}$ in (10) are bounded below, define

$$
c_{k}=\inf \left\{p_{k}(t) \mid t \geqq a\right\}, \quad q(\lambda)=\sum_{k=0}^{n}(-1)^{k} c_{k} \lambda^{2 k} .
$$

Assume also $c_{n}>0$ and $q(\lambda)=0$ has no zero or purely imaginary roots. This condition gives that (10) is nonoscillatory, i.e., no solution has a pair of $\boldsymbol{n}$-fold zeros [see p. 129 of Glazman (1965)]. Thus by Coppel, p. 45, a principle solution exists. Let

$$
A^{+}=\left\{x \mid L(x)=0 \quad \text { and } \quad x^{(i)} \in \mathscr{L}_{2}[a, \infty), \quad i=0, \cdots, n\right\} .
$$

Under these conditions, one of the conclusions of Anderson and Lazer's theorem is that $\operatorname{dim} A^{+} \geqq n$.

For $c_{i} \geqq 0, i=1, \cdots, n-1, \operatorname{dim} A^{+} \geqq n$ follows from part (i) of Theorem 1 since we must have $c_{n}>0, c_{0}>0$ and from (12) we see that $(y, z) \in Q$ implies that $x^{(i)} \in \mathscr{L}_{2}[a, \infty), i=0, \cdots, n$.

Finally, we note that if $v_{1}, \cdots, v_{n}$ are the $n$ linearly independent members of $A^{+}$as constructed in Anderson and Lazer, then $(Y, Z)$ is a 
principal solution of (2) where the $i$ th column $\left(y_{i}, z_{i}\right)$ is given by (11) with $x=v_{i}$. From Anderson and Lazer, we have that $Y(a)$ is a non-singular diagonal matrix and

$$
Y(t)=\operatorname{limit}_{n \rightarrow \infty} Y_{n}(t)
$$

where $\left(Y_{n}, Z_{n}\right)$ is certain solution of (2) with $Y_{n}(n)=0$. It is sufficient that $Y_{n}(a)=I$. By p. 44 of Coppel, $Y_{n}$ is unique and the limit in (18) is principal.

\section{References}

C. D. Ahlbrandt (1972), 'Principal and antiprincipal solutions of self-adjoint differential systems and their reciprocals', Rocky Mountain J. Math. 2, 169-182.

C. D. Ahlbrandt (1976), 'The question of equivalence of principal and coprincipal solutions of self-adjoint differential systems', Illinois $J$. Math. 20, 72-81.

L. R. Anderson and A. C. Lazer (1970), 'On the solutions of linear self-adjoint differential equations', Trans. Amer. Math. Soc. 152, 519-530.

W. A. Coppel (1971), Disconjugacy (Springer-Verlag Lecture Notes in Mathematics, vol. 220, Berlin).

I. M. Glazman (1965), Direct methods of qualitative spectral analysis of singular differential operators (Israel program for scientific translation, Jerusalem).

D. B. Hinton (1972), 'Limit point criteria for differential equations', Canad. J. Math. 24, 293-305.

D. B. Hinton (1973), 'Limit point criteria for positive definite fourth-order differential operators', Quart. J. Math. Oxford (2) 24, 367-376.

W. T. Reid (1971), Ordinary differential equations (Wiley, New York).

Mathematics Department,

The University of Tennessee,

Knoxville, 37916,

Tennessee, U.S.A. 